\title{
DETECTION AND MITIGATION OF MAC SPOOFING
}

\author{
Hammal Mangal ${ }^{1}$, Muhammad Usman ${ }^{2}$ \\ ${ }^{1,2}$ Alhamd Islamic University, Quetta \\ engrhammal@outlook.com
}

\begin{abstract}
Wireless Local Area Network (WLAN) are generally utilized and getting more in number step by step because of the simplicity of spread signs, quality, and quality. WLAN is additionally simple to actualize in any association. Without any difficulty of utilization, the remote system is likewise simple to produce or bargain because of some shortcoming. Macintosh satirizing is one of the provoking system to be maintained a strategic distance from Macintosh satirizing because conveyable due to devices used to produce the MAC address of system card on the product level. Approval with MAC address got dubious. To beat this issue, we proposed a structure in which client approval process completed by getting three one of kind boundaries of the machine, get hashed and contrasted and the database.
\end{abstract}

Keywords—Detection and Mitigation. MAC Spoofing.

\section{INTRODUCTION}

Wireless Local Area Network (WLAN) has picked up acclaim by its current various application and execution ease. It has been evaluated that WI-FI market will develop by worth USD 14.8 Billion of every 2015 to USD 33.6 Billion by 2020 . It depends on the IEEE 802.11 principles and has contained some client machines [1]. Also, a passageway has furnished availability to the client's machine with one another and webs displayed in Figure 1.

Ongoing years have seen that regular utilization of WLAN likewise pulled in by the assailants. The signs of remote system are open in nature, so anybody in range can stiff the sign or bundles moving noticeable all around. Remote systems have utilized MAC address to recognize the client's gadget because of its special property [2].

Also Media Access Control (MAC) address has a special identifier of each machine and MAC address has been doled out to Network Interface Card (NIC) for correspondence over the information connect [3]. Similarly, it has been utilized for correspondence by Ethernet convention with no limitation of what application convention has a should be utilized on head of it.

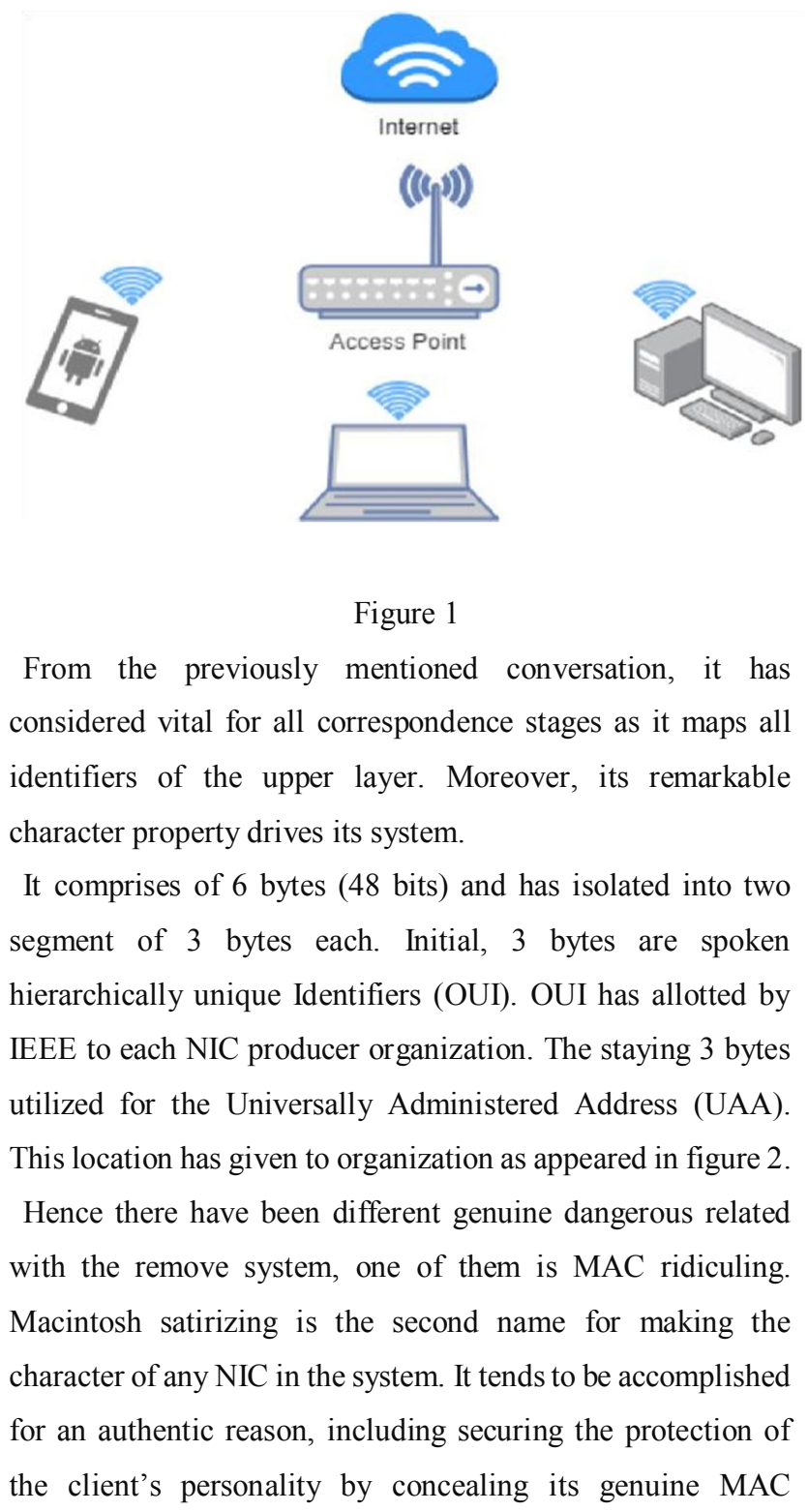


ISSN: 2708-7123 | Volume-01, Issue Number-02 | June-2020

LC INTERNATIONAL JOURNAL OF STEM

Web: www.logicalcreations.org/stem | www.lcjstem.com | DOI: https://doi.org/10.47150

address in the system. This strategy additionally utilized by aggressors to perform different sort of assaults, for example customer caricaturing, Man in the center (MITM), Access point mocking, Daniel of services [4]. The fundamental target of MAC ridiculing is concealing the movement or sidestep get to control list. Be that as it may, MAC address can without much of a stretch get by sniffing the Wi-Fi systems. Numerous devices have excited for such a reason. In Linux based framework and windows OS based machines. Air crack-ng has utilized. In the wake of picking up the MAC address assailant can alter its MAC address with real MAC address to get to the framework however windows OS based machines and Linux based machines, MacMakeup [5] and Macchanger [6] have furthermore used individually.

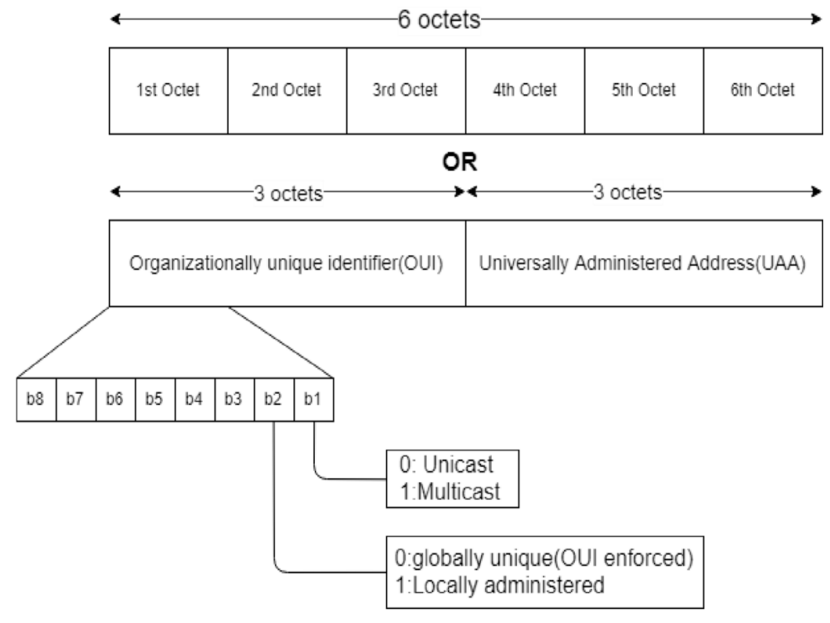

Figure 2

Problem Statement:

From the previously mentioned conversation MAC caricaturing is hurtful and the purpose behind numerous extreme assaults. In the creators have tended to this issue by breaking down the sign quality. They have utilized different Access focuses to catch signal quality, however if there should be an occurrence of an authentic client and assailant are on a similar separation, the individual strategy has not been viewed as valuable. In this way, in this investigation, we have proposed the structure to control customer caricaturing assault that has generally used to sidestep the MAC filtration or Access control records.

\section{RELATED WORK}

Macintosh satirizing is a significant issue for security specialists in view of its serious assaults. This segment includes some agent techniques.

In the creators have been portrayed the strategy to identify the parodying by signal quality to identify the parodying by signal quality originating from the authentic hub and furthermore from the assailant hub. They have identified the nearness of an aggressor in the system by utilizing the proposed strategy while the constraint has the two hubs ought to be fixed. Moreover, they have utilized many air screens.

In the creators have proposed cryptographic encryption procedures. These methods have used to stop to stop the MAC parodying. In any case, the encryption plans over-burden both the Access point and the hub.

Have introduced a location component for grouping based MAC satirizing. Each MAC address has a groping number filed. This number has thought to be incremented by one for each cordial information and the board outline. Any unusual hole between the casings from similar MAC address can be considered as a sign of mocking. Be that as it may, this methodology can prompt a high pace of bogus positive in view of lost or copy parcels.

\section{ATTACKER MODEL}

In an assailant model, we have completed an assault situation wherein an aggressor can sidestep the MAC filtration on the switch. In this situation, we have a passageway and a few clients. In passage, a MAC filtration has been executed which permits simply genuine clients to associate with the passageway. On the off chance that a hub with exception of genuine client to interface, the passage won't permit the nonauthentic client to get an association as appeared in figure 3 .

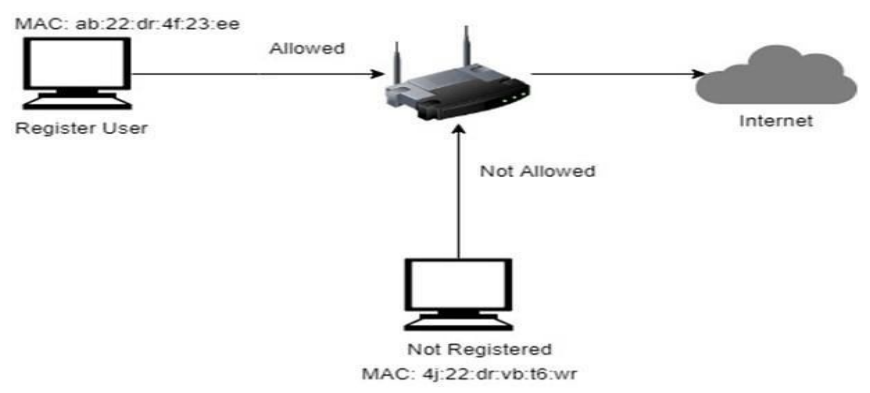

Figure 3 
ISSN: 2708-7123 | Volume-01, Issue Number-02 | June-2020

LC INTERNATIONAL JOURNAL OF STEM

LC-JS'TEM

Web: www.logicalcreations.org/stem | www.lcjstem.com | DOI: https://doi.org/10.47150

In an assailant model, we have completed an assault situation wherein an aggressor can sidestep the MAC filtration on the switch. In this situation, we have a passageway and a few clients. In passage, a MAC filtration has been executed which permits simply genuine clients to associate with the passageway. On the off chance that attempted to interface, the passage won't permit the non-authentic clients to get an association as appeared in figure 4 maps the exact scenario.

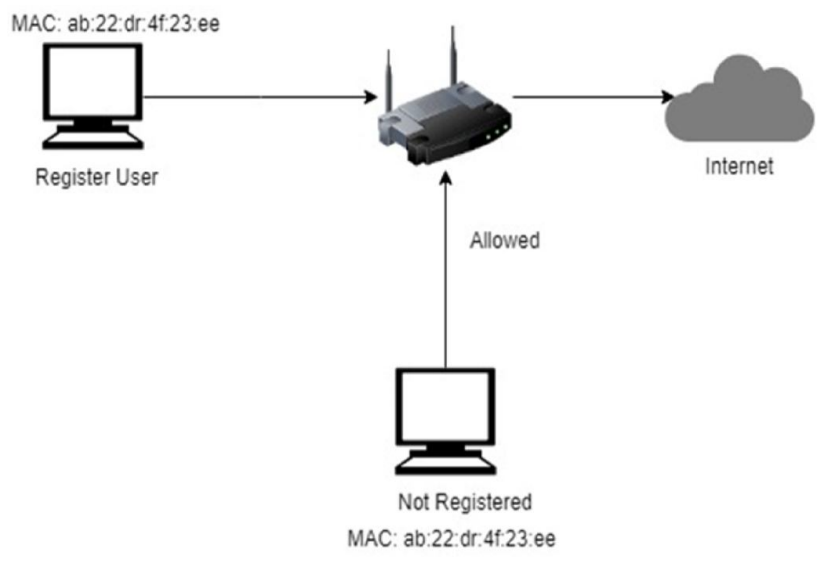

Figure 4

\section{PROPOSED FRAMEWORK}

A system has proposed in this paper to control the MAC parodying in the system. Prior to this, just MAC address has utilized for the approval reason and whenever ridiculed MAC address attempts to sidestep the approval, switch thinks of him as a real clients and permit the administration.

In most recent years, for the approval reason, MAC address has been utilized as it has extraordinarily assigned to each machine on the planet. Be that as it may, later apparatuses make it conceive to change the MAC address on the Software level [7-9]. Hence, a solitary boundary isn't sufficient to verify in the system. The principle challenge was to discover different boundaries which are interesting in each framework and furthermore ought not to be anything but difficult to change these boundaries on the product level. In this investigation, out of numerous boundaries the three boundaries that we have picked incorporate MAC address, process ID and battery sequential number [10].

As effectively characterized MAC address is one of a kind for each framework and primarily utilized for approval reason.
Other than that, each process has an extraordinarily characterized ID called pid which can be utilized for the approval reason. The third and last one is Battery sequential number. Each battery forced a sequential number during the assembling methodology. Battery sequential number is exception and can be utilized for approval reason.

Thus in this proposed framework when an authentic and enrolled client will attempt to associate the principal framework will get MAC, Process ID, and Battery sequential number of the machine, link them and compute the hash. In the wake of getting hash the framework will analyse hash in its database. On the off chance that the hash found in a database framework will permit the client to get associated in the system. Be that as it may, if the hash isn't found or coordinated, the framework won't permit the client to get associated as appeared in figure 5. On another hand, if the client is new and needs to be give MAC address, Processor ID, and Battery sequential number to the Network overseer at that point organize executive will connect them and compute the hash. In the wake of figuring hash, it stores in the database and has permitted the client for network. The whole process is shown I figure 6.

\section{CONCLUSION}

Macintosh ridiculing has used to sidestep the approval procedure of MAC filtration or Access control procedure records. Macintosh address have one of kind for each framework yet can without much of a stretch be caricature by various devices. So approval with just MAC address has not been viewed as adequate. Our proposed system Make approvals increasing solid even MAC address get ridiculed by actualizing hash calculations.

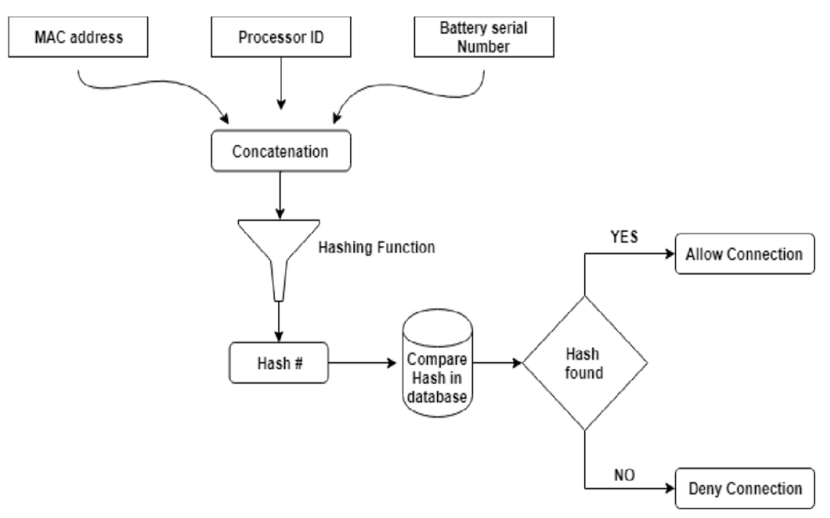


ISSN: 2708-7123 | Volume-01, Issue Number-02 | June-2020

LC INTERNATIONAL JOURNAL OF STEM

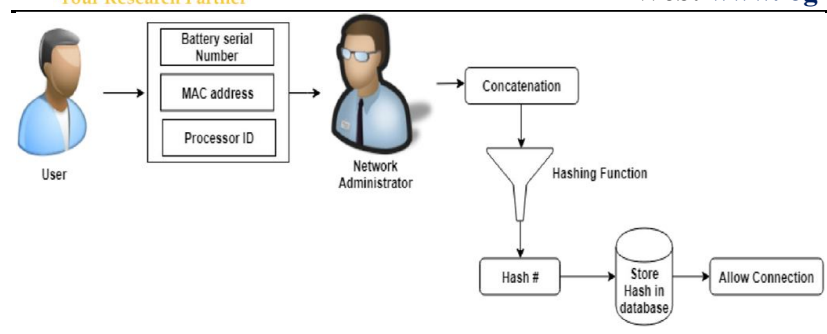

\section{REFERENCES}

1. Online Accessible

https://www.marketsandmarkets.com/Market-

Reports/global-wi-firmket994.html

2. R. a. T. S. a. B. D Bansal,

Non cryptographic method of MAC spoof detection in wireless LAN Networks,

2008 ICON 2008. $16^{\text {th }}$ IEEE international

conference on, pp. $1-62008$.

3. S. Whalen, an introduction to arp snooping in node99 online document April, 2001.

4. G. a. P. U. a. T. P. Lackner, Combating Wireless LAN MAC-layer Address Spoofing with Fingerprint methods.

J network Security vol. 9. No. 2 pp.164-172, 2009.

5. C. a. B. A. a. D. F. Z. a. A.-N. A. a. Z. K. Benza \{\” i) d,

Intelligent detection of MAC Spoofing attack in 802.11 networks in Proceeding of the $17^{\text {th }}$ international conference on Distributed Computing and networking, 2016

6. V. a. A. V. a. H. D. Nagarajan

Using power hoping to counter MAC spoof attacks in WLAN,

Commutations and Networking Conference

(CCNC), $20107^{\text {th }}$ IEEE, pp. 1-5, 2010.
7. SZ Iqbal, K. S. (2020). Improving Software Cost Estimation With Function Points Analysis Using Fuzzy Logic Method. LC International Journal of STEM, 1(1), 12-21.

8. Online Available:

https://www.aircrack-ng.org

9. Online Available:

https://www.gorlani.com/software/mmk

10. Online Available:

https://github.com/alobbs/macchanger

11. Y. a. T. K. a. C. G. a. K. D. a. C. A Sheng Detecting 802.11 MAC layer spoofing using received signal INFOCOM 2008, The $27^{\text {th }}$ Conference on computer Communication, IEEE pp. 1768---1776, 2008

12. P. a. V. S. a. B. A. Bahl

Wireless internet access in public places. Communications, 2001. ICC 2001. IEEE International Conference on vol. 10, pp. 32713275,2001

13. F. a. C. T.-c. Guo, Sequence number based MAC address spoof detection International workshop on Recent Advances in intrusion Detection, pp. 309-329, 2005.

14. G. a. J. C. Joshua Wright, Detecting wireless LAN MAC address spoofing Cisco Certified Network Associate 2003.

15. T. Okada

Processor with a function to prevent illegal execution of a program, an instruction executed by a processor and a method of preventing illegal execution of a program. 
Patent US Patent 6,704,872,9 mar 2004.

16. Y.-W. a. W. R. a. H. S. Chung,

Wireless communication device having battery

authentication and associated method.

Patent US Patent App. 11/053,453, 2006.

17. A. a. M. R. a. S. B. a. W. N. C. Sanzgiri

Method to secure 802.11 traffic against MAC

address spoofing.

US Patent US 2006/0114863 AI, jun \# “ 1”, 2006. 